\title{
openheart Annular sizing using real-time three-dimensional intracardiac echocardiography-guided trans-catheter aortic valve replacement
}

Alejandro Rendon, ${ }^{1}$ Tahir Hamid, ${ }^{2}$ Gajen Kanaganayagam, ${ }^{3}$ Devinda Karunaratne,${ }^{2}$ Vaikom S Mahadevan ${ }^{2,4}$

To cite: Rendon A, Hamid T, Kanaganayagam G, et al. Annular sizing using real-time three-dimensional intracardiac echocardiography-guided trans-catheter aortic valve replacement. Open Heart 2016;3:e000316. doi:10.1136/openhrt-2015000316

- Additional material is available. To view please visit the journal (http://dx.doi.org/ 10.1136/openhrt-2015000316).

Received 17 September 2015 Accepted 25 January 2016

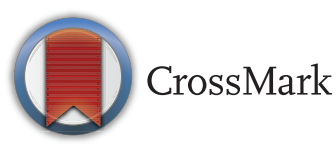

\footnotetext{
${ }^{1}$ Siemens UK Itd, London, UK ${ }^{2}$ Manchester Royal Infirmary, University of Central Manchester NHS foundation Trust, Manchester, UK ${ }^{3}$ Royal Brompton Hospital, London, UK

${ }^{4}$ University of California, San Francisco, USA
}

Correspondence to Dr Vaikom S Mahadevan; vsmkm@yahoo.co.uk

\section{ABSTRACT}

Objective: Transcatheter aortic valve replacement (TAVR) has been established as an alternative therapy for patients with severe aortic stenosis who are unfit for the surgical aortic valve replacements. Pre and periprocedural imaging for the TAVR procedure is the key to procedural success. Currently transesophageal echocardiography (TOE), including real-time threedimensional (RT-3D) imaging TOE, has been used for peri-interventional monitoring and guidance for TAVR. We describe our initial experience with real-time threedimensional intracardiac echocardiography (RT-3DICE), imaging technology for the use in the TAVR procedure. Methods: We used RT-3DICE using an ACUSON SC2000 2.0v (Siemens Medical Solution), and a 10F AcuNav V catheter (Siemens-Acuson, Inc, Mountain View, California, USA) in addition to preoperative multislice CT (MSCT) in total of five patients undergoing TAVR procedure.

Results: Aortic annulus and sinus of valsalva diameters were measured using RT-3DICE. Aortic valve measurements obtained using RT-3DICE are comparable to those obtained using MSCT with no significant difference in our patients.

Conclusions: This small study of five patients shows the safe use of RT-3DICE in TAVR Procedure and may help the procedures performed under local anaesthesia without the need for TOE.

\section{INTRODUCTION}

Transcatheter valve therapies are being increasingly used in the treatment of valvular heart disease. Transcatheter aortic valve replacement (TAVR) has been adopted as an alternative method of treatment for patients with severe aortic stenosis (AS) who are deemed unfit for open valve surgery. Pre and periprocedural imaging is pivotal for accurate valve sizing, deployment and assessing for postprocedural aortic and paravalvular regurgitations and other complications. The valve sizing is one of the most important factors for this procedure to be successful; therefore

\section{KEY QUESTIONS}

What is already known about this subject?

- Pre and periprocedural imaging is essential for accurate valve sizing, deployment and to assess for procedural complications during transcatheter aortic valve replacement (TAVR). Two-dimensional transoesophageal echocardiogram (TOE) tends to underestimate the aortic valve annulus diameter and can have significant impact on the selection of the prosthetic valve size to implant. Moreover use of TOE requires use of general anaesthesia.

What does this study add?

- We describe the use of real-time threedimensional intracardiac echocardiography (RT-3D ICE), AcuNav catheters for accurate aortic root dimensions in patients undergoing TAVR and compare sizing using this technique against that obtained using multislice CT. This is the first such reported study to the best of our knowledge.

How might this impact on clinical practice?

- This study shows that RT-3DICE is a safe and effective technique in assessing valve size and reduces the need for TOE in this era of TAVR, where more procedures are performed under local anaesthesia.

accurate measurements of the aortic root are needed preprocedure. A variety of imaging techniques, ${ }^{1}$ including multislice CT (MSCT) has been described as the gold standard to assess aortic root and annulus dimensions preprocedure, two-dimensional (2D) echocardiography, three-dimensional (3D) echocardiography and the use of transesophageal echocardiography (TOE) during the procedure for deployment of the valve. Previous studies ${ }^{1}$ have demonstrated that 2D TOE tends to underestimate the aortic valve annulus diameter, with a modest correlation 
Table 1 3D-ICE results, linear and planimetry measurements were obtain in PLAX and SAX (equivalent to 130-150 TOE views)

\begin{tabular}{|c|c|c|c|c|c|c|c|}
\hline \multirow[b]{2}{*}{ Case } & \multicolumn{4}{|l|}{ RT-3D ICE } & \multicolumn{3}{|l|}{ MSCT } \\
\hline & $\begin{array}{l}\text { Ao annulus } \\
\text { (cm) }\end{array}$ & $\begin{array}{l}\text { Ao sinus } \\
\text { (cm) }\end{array}$ & $\begin{array}{l}\text { Ao sinus }(\mathrm{cm}) \\
\text { SAX }\end{array}$ & $\begin{array}{l}\text { Annulus } \\
\text { area }\end{array}$ & $\begin{array}{l}\text { Ao annulus } \\
\text { (cm) }\end{array}$ & $\begin{array}{l}\text { Ao sinus } \\
\text { (cm) }\end{array}$ & $\begin{array}{l}\text { Annulus } \\
\text { perimeter } \\
\left(\mathrm{mm}^{2}\right)\end{array}$ \\
\hline 1 & 2 & 3.2 & $3.0 \times 2.0$ & 500 & $3.0 \times 2.2$ & $3.2 \times 2.9$ & 513 \\
\hline 2 & 2.4 & 3.1 & $3.0 \times 3.0$ & 510 & $2.3 \times 2.2$ & $3.1 \times 3.0$ & 530 \\
\hline 3 & 2.2 & 3 & $3.3 \times 2.2$ & 527 & $3.3 \times 2.2$ & $3.7 \times 3.0$ & 554 \\
\hline 4 & 2.2 & 3.6 & $3.0 \times 3.0$ & 498 & $2.8 \times 2.1$ & $3.3 \times 3.2$ & 513 \\
\hline 5 & 2.3 & 3.6 & $3.6 \times 3.2$ & 580 & $3.0 \times 2.6$ & $3.7 \times 3.2$ & 629 \\
\hline
\end{tabular}

MSCT,multislice CT; RT-3D ICE,real-time three-dimensional intracardiac echocardiography; TOE, two-dimensional transoesophageal echocardiogram.

between the 2D measurements and the MSCT. Underestimation of the diameters are most likely due to the fact that measurements performed in 2D were at a tangent cut across the aortic root. In fact the aortic root is not completely circular at any point, and the shape of the aortic annulus is more elliptical with a maximum and minimum diameters. Routine 2D measurements of the aortic root or annulus by any of these techniques will depend on the plane and angulation, which will have a significant impact on the selection of the prosthetic valve size to implant. We describe the use of realtime three-dimensional intracardiac echocardiography (RT-3D ICE), AcuNav V catheters for accurate aortic root dimensions in patients undergoing TAVR and compare sizing using this technique against that obtained using MSCT. ICE has been used with added

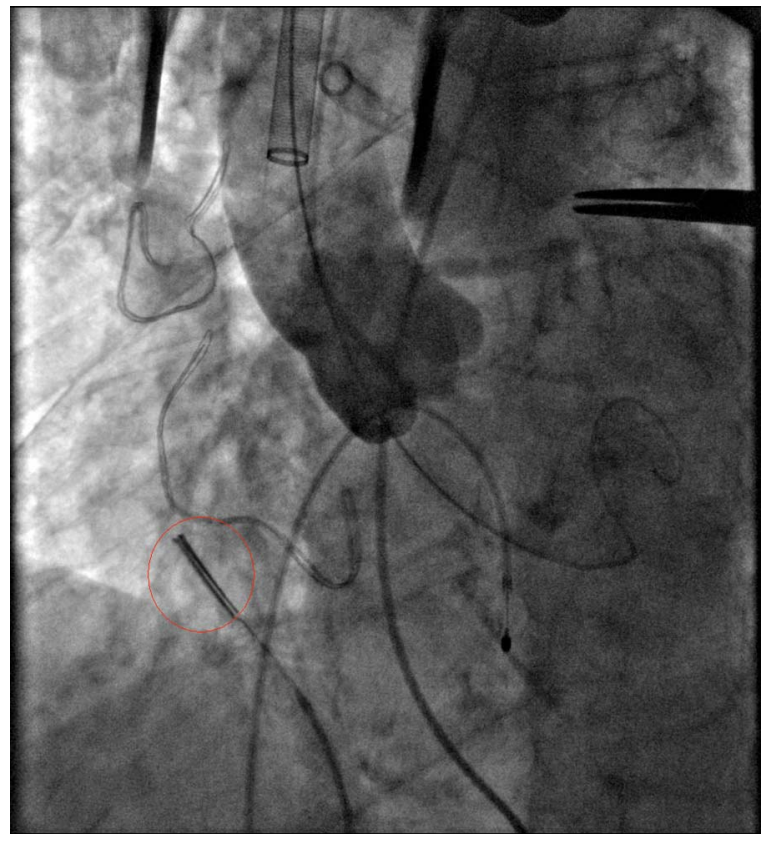

Figure 1 The image shows intracardiac echocardiography (ICE) probe in a TAVI procedure. TAVI, transcatheter aortic valve implantation. advantage in guiding device closure of interatrial shunts and electrophysiological ablation procedures. ${ }^{2}$

\section{Case reports}

Five patients (3 females) with severe AS underwent TAVR procedure using RT-3DICE using an ACUSON SC2000 2.0v (Siemens Medical Solution), and a $10 \mathrm{~F}$ AcuNav $\mathrm{V}$ catheter (Siemens-Acuson, Inc, Mountain View, California, USA) in addition to preoperative MSCT. Aortic valve measurements obtained using RT-3DICE are MSCT and shown in table 1. Real-time monitoring (figure 1 and see online supplementary video file 1) was performed with an Acunav V (volume mode using a $90 \mathrm{~cm}, 10-\mathrm{F}$ phased-array) catheter capable of articulating $160^{\circ}$ in four directions. A 3D imaging volume size of $22^{\circ} \times 90^{\circ}$ was acquired at $6-8 \mathrm{MHz}$ frequency. The ICE catheter was inserted via a $12 \mathrm{~F}$ right femoral vein sheath and placed at the roof of the right atrium anterior to the aortic valve (figures 1 and 2). Three Edwards Sapien XT valves (size $26 \mathrm{~mm}$ ) were implanted and two Edwards Sapien S3 valves (size $26 \mathrm{~mm}$ ). There was no procedural or 30 day mortality and no procedure-related complications. All patients had concomitant TOE except one who had a large hiatus hernia primarily to assess the presence and severity of aortic or paravalvular regurgitation. Four of the five patients underwent TAVR implantation via the transfemoral route and one patient via the transaortic route. Aortic annulus and sinus of valsalva diameters were measured using RT-3DICE. An independent radiologist blinded to RT-3DICE and TOE measurements performed the MSCT measurements. Post-TAVR, one patient had mild paravalvular aortic regurgitation and quantification was similar on TOE and RT-3DICE.

\section{DISCUSSION}

TAVR is increasingly used as the procedure of choice for patients who are considered as high risk for surgical aortic valve replacement, however more recent studies are also looking at patients, who are at intermediate risk for aortic valve surgery towards being treated with a transcatheter aortic valve implantation procedure. ${ }^{2}$ 
Figure 2 Pre-TAVI procedure annular area. 3D-ICE annulus area by planimetry versus MSCT annulus area. TAVI, transcatheter aortic valve implantation; MSCT, multislice CT; 3D-ICE, three-dimensional intracardiac echocardiography.

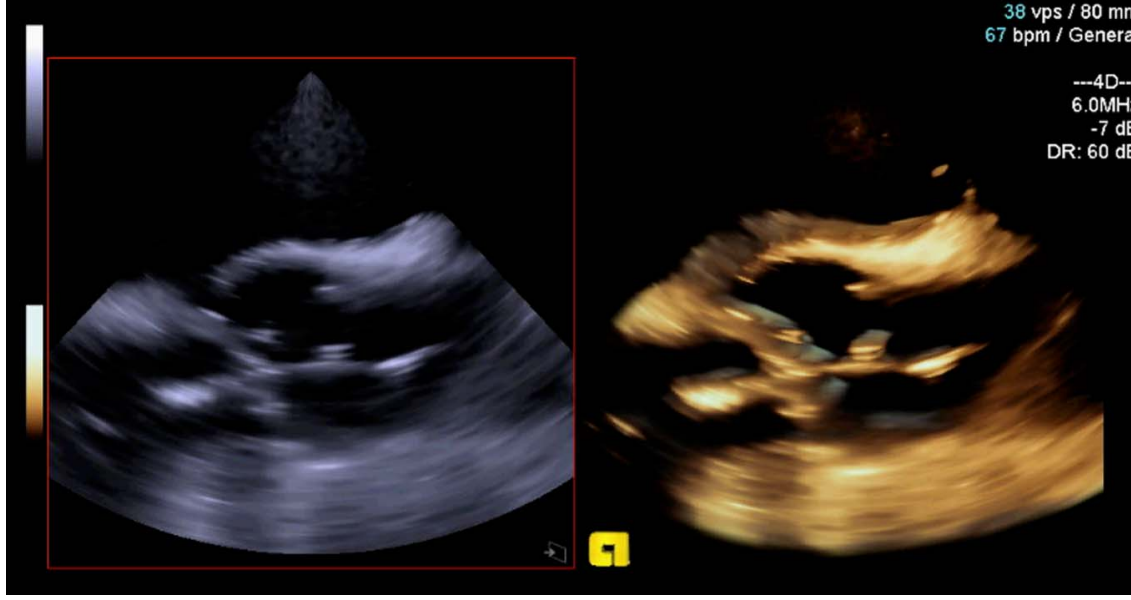

TAVR is a complex procedure requiring appropriate patient selection and valve choice. The Experience with RT-3DICE is currently limited $^{3}$ and has been described in the closure of septal defects. As the trend for early discharge following TAVR deployment is increasing more procedures are being performed under local anaesthesia (LA). ${ }^{4}$ For patients undergoing procedure under LA, TOE imaging is uncomfortable and not desirable. RT-3DICE would certainly play an important role in these patients, since only transvenous access is required and would not exclude patients with severe peripheral vascular disease who undergo TAVR using alternate access. This is the first reported study of use of RT-3DICE in TAVR. Our study shows this is feasible and safe. Aortic valve measurements obtained using RT-3DICE are comparable to those obtained via MSCT in our five patients. Newer RT-3DICE catheters with a larger imaging volume size are likely to produce even better images and data for valve area measurements.

\section{LIMITATIONS}

This is a small study in five patients. A larger study is required to confirm this data. There is also likely to be an operator learning curve for the use of the RT-3DICE catheter. The incremental value of RD-3DICE may be limited for sizing as it is usually established before the procedure however in patients in whom such imaging is not definitive or possible, RT-3DICE offers a further option for sizing.

\section{CONCLUSION}

This study shows that RT-3DICE is a safe and effective technique in assessing valve size and looking at aortic valve and paravalvar leak in patients undergoing TAVR procedures.

Competing interests None declared.

Provenance and peer review Not commissioned; internally peer reviewed.

Data sharing statement No additional data are available.

Open Access This is an Open Access article distributed in accordance with the Creative Commons Attribution Non Commercial (CC BY-NC 4.0) license, which permits others to distribute, remix, adapt, build upon this work noncommercially, and license their derivative works on different terms, provided the original work is properly cited and the use is non-commercial. See: http:// creativecommons.org/licenses/by-nc/4.0/

\section{REFERENCES}

1. Zamorano JL, Gonçalves A, Lang R. Imaging to select and guide transcatheter aortic valve implantation. Eur Heart $J$ 2014;35:1578-87.

2. Fontes-Carvalho R, Sampaio F, Ribeiro J, et al. Three-dimensional intracardiac echocardiography: a new promising imaging modality to potentially guide cardiovascular interventions. Eur Heart $J$ Cardiovasc Imaging 2013;14:1028.

3. Cunnington $\mathrm{C}$, Hampshaw SA, Mahadevan VS. Utility of real-time three-dimensional intracardiac echocardiography for patent foramen ovale closure. Heart 2013;99:1789-90.

4. Fröhlich GM, Lansky AJ, Webb J, et al. Local versus general anesthesia for transcatheter aortic valve implantation (TAVR)systematic review and meta-analysis. BMC Med 2014;12:41. 